\title{
Histological and structural analysis of Actinia equina L. (Cnidaria: Anthozoa)
}

\author{
J. R. Gadelha***, F. Morgado* and A.M.V.M. Soares* \\ * CESAM \& Departamento de Biologia \\ ** CAPES Foundation, Ministry of Education of Brazil, Brasília - DF 70040-020, Brazil
}

The Anthozoa class is composed solely by marine benthic consumers, some with adaptations that enable them to inhabit even the most extreme habitats, like hydrothermal vents, the polar seas, and even whale falls. Anthozoans, such as corals and anemones, are important modulators of marine habitats in the coral reef communities, being also ecologically relevant due to their abundance and the trophic level they occupy. One of these is Actinia equina, the most conspicuous species in tidal pool communities in European shores, with a broad range of distribution from the North Sea to the Mediterranean and Aegean seas and South Africa. Histological and structural analyses allows for a more detailed biological and ecological study of these organisms, including their reproductive and metabolic behaviour [3]. This work describes a morphologic and histologic analysis of A. equina, with these endpoints for the first time. Organisms, collected to the natural environmental were processed using a paraffin-embedding technique. Further, samples were processed following by Hematoxilin and Eosin ( $\mathrm{H} \& \mathrm{E})$ standard procedures with adaptations to Actiniidae conditions (the fixation time was 96 hours due to $98 \%$ of water body in these organisms). Results obtained confirm that, when compared to higher level animals [1,2], the Anthozoa are very simply organized (Figure 1). The body is built up of sheets of tissue (Figure 2 and 3), on the surface of each there is an epithelium, resting upon a middle layer of fibrous mesoglea. This generally contains no cellular elements other than some scattered amoebocytes and locally, genital cells (Figure 2). The aim of this work was to contribute to a new anatomic and histological baseline for these sea anemones family Actiniidae. In order to identify several taxonomic characteristics based on reproductive structures. The methods applied were standard to identify several structures that acid-basic groups ligands, such as phosphate of DNA, collagen and other nuclear proteins. The results obtained were the basic cnidarian structures such as tentacular and mesenteric anatomy. The reproductive structures are show in detail with septal filaments and mesenteric (Figure1). These structures is taxonomically relevant and into the own family (Actiniidae) could following the same patterns. The male and female A. equina mesenteries are different. The mainly conclusion of this study was that the male and female gonads can appear simultaneously in the same individual, or that it can have no defined sex.

\section{References}

1. Carter M.A. and Miles J., Gametogenic Cycles and Reproduction in the Beadlet Sea-Anemone Actinia equina (Cnidaria, Anthozoa). Biological Journal of the Linnean Society 36: 129-155, 1989

2. Chia F.S. and Rostron M.A., Some Aspects of Reproductive Biology of Actinia equina CnidariaAnthozoa. Journal of the Marine Biological Association of the United Kingdom 50: 253, 1970

3. Watts, P.C. et al., An analysis of the nematocysts of the beadlet anemone Actinia equina and the green sea anemone Actinia prasina. Journal of the Marine Biological Association of the United Kingdom 80: 719-724, 2000

Acknowledgments: This work was supported by the Portuguese Foundation for the Science and Technology - Portugal and FEDER funds, through the Projects: PTDC/MAR/464729/2006 and FCT/CNPq (Brazil), Project 6818, Programme 19/ 004. 

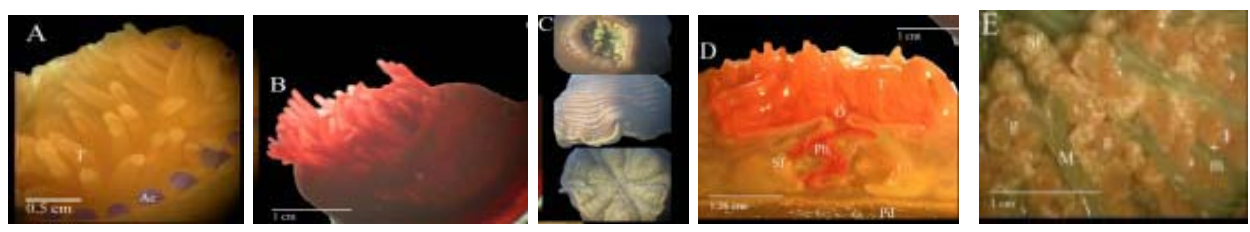

Figure 1. Macroscopic aspect of A. equina. A - Yellow morphology capitullum with visible achoragi. B - Red green morphology, semi-expanded. C - Preserved whole individual; Up-down: oral, lateral and bottom views. D - Longitudinal section. E - Cross section at the coelenterons region, with septal filaments and fertile mesenteries visible.

Abbreviations: Ac, Achrorragus (in A); g, gonad area of the mesentery; M, Complete mesentery; $\mathrm{m}$, incomplete mesentery (in E); O, oral opening; Ph, actinopharinx; Pd, Pedal disk (in D).

Figure 2. Hematoxylin and eosin stained serial sections of $A$. equina. A, Tentacle; B, Actinopharynx; C, Pedal disk; D, Column; E, Peristome; F, Cross section of column gastrodermis; G, Detail of column mesoglea; $\mathrm{H}$, Infertile mesentery.

Abbreviations: Am, Ameobocyte; $\mathrm{Ct}$, Cilliary tract; Dt, Cnidoglandular tract; GC, Agranular gland cell; GGC, Granular gland cell; M, Mucous cell; S, spirocyst; Sb, Spiroblast. Scale bar: 0.01 $\mathrm{mm}$.
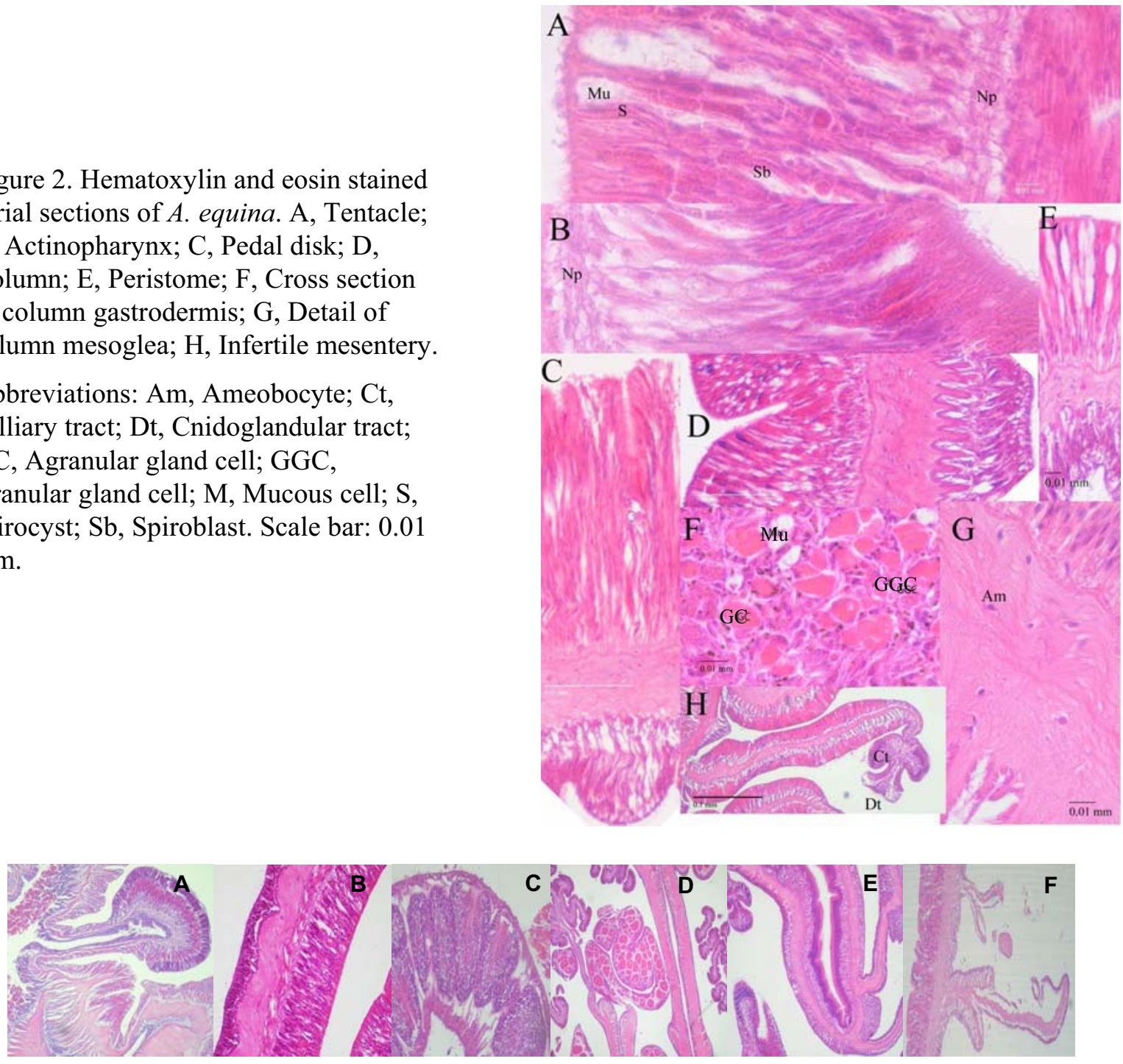

Figure 3. Microphotographs of A. equina sections, Hematoxylin and eosin stained. A- Diffuse gastrodermic marginal sphincter muscle (Sm) and acrorhagus; B - Transverse section of the tentacle; C - Mature sperm packets; D - Female gonads in three different mesentery cycles; E - Siphonoglyph region of the Actinopharinx; F - Basiliary muscles. 